\title{
Control óptimo de la concentración de dióxido de carbono en una columna de absorción empacada mediante un modelo de red neuronal artificial
}

\author{
Optimal control of the composition of carbon dioxide in the absorption column packed \\ by means of a model of artificial neural network
}

\author{
Iván L. Osorio L.' \& Venancio S. Navarro R. \\ 'Facultad de Ingeniería Química, Universidad Nacional del Centro del Perú. \\ Email: iosorio1986@yahoo.com
}

\section{RESUMEN}

El objetivo de este trabajo de investigación fue realizar el control óptimo de la composición de dióxido de carbono en la columna de absorción empacada del Laboratorio de Operaciones y Procesos Unitarios de la Facultad de Ingeniería Química mediante un modelo de red neuronal artificial. Un modelo de red neuronal artificial con una capa de entrada, una capa escondida y una capa de salida fue desarrollado, entrenado con el algoritmo de retropropagación y validado experimentalmente para un proceso de absorción con reacción química. Asimismo, siguiendo el mismo procedimiento fue implementado el modelo del neurocontrolador (modelo inverso de la red neuronal artificial). El trabajo experimental fue realizado en una columna de absorción de vidrio pyrex de 1.15 metros de altura y 8.5 centímetros de diámetro interno, empacada con anillos Raschig de vidrio de 20 milímetros y opera con un rango en el flujo de gas de 12 a $16 \mathrm{~L} / \mathrm{min}$ y un rango en el flujo de líquido de 0.8 a $2.8 \mathrm{~L} / \mathrm{min}$. La variable controlada fue la concentración de dióxido de carbono a la salida de la columna de absorción en un rango de 4 a $8 \% \mathrm{~V}$ y la variable manipulada fue la velocidad de flujo de entrada de la solución de hidróxido de sodio $0.11 \mathrm{M}$ en un rango de 0.8 a $2.8 \mathrm{~L} / \mathrm{min}$. Para contrastar la hipótesis el neurocontrolador fue probado experimentalmente estableciéndose diferentes valores de referencia (set points) y el control óptimo de la columna de absorción demostró ser realizable suministrando una adecuada acción de control para alcanzar los valores de referencia deseados.

\section{ABSTRACT}

The objective of this research work was perform the optimal control of the composition of carbon dioxide in the absorption column packed of the Laboratory of Unit Operations and Processes of the Faculty of Chemical Engineering by means of a model of artificial neural network. A model of artificial neural network with an input layer, a hidden layer and an output layer was developed, trained with the backpropagation algorithm and validated experimentally for a process of absorption with chemical reaction. In addition, following the same procedure was implemented the model of the neurocontroller (inverse model of the artificial neural network). The experimental work was carried out in an absorption column of pyrex glass of 1.15 meters in height and 8.5 centimeters of internal diameter, packed with glass Raschig rings of 20 millimetres and operates with a range in the gas flow of 12 to $16 \mathrm{~L} / \mathrm{min}$ and a range in the liquid flow rate of 0.8 to $2.8 \mathrm{~L} / \mathrm{min}$. The controlled variable was the concentration of carbon dioxide out of the absorption column in the range 4 to $8 \% \mathrm{~V}$ and the manipulated variable was the speed of input stream of the sodium hydroxide solution in $0.11 \mathrm{M}$ in a range of 0.8 to $2.8 \mathrm{~L} / \mathrm{min}$. To contrast the hypothesis the neurocontroller was tested experimentally establishing different reference values (set points) and the optimal control of the absorption column proved to be feasible by providing adequate control action to achieve the desired reference values.

\section{Palabras clave | Key words:}

control óptimo de la composición de dióxido de carbono, columna de absorción empacada, modelo de red neuronal artificial.

optimal control of the composition of carbon dioxide, absorption column packed, model of artificial neural network. 


\section{INTRODUCCIÓN}

La absorción es uno de los métodos para eliminar el dióxido de carbono (CO2) en el gas natural y en los gases de combustión, para que no ingrese a la atmósfera y contribuya al calentamiento global. En la absorción química el gas que se va a eliminar reacciona con el solvente y queda en solución, como es el caso de la eliminación de CO2 por reacción con hidróxido de sodio ( $\mathrm{NaOH})$.

La absorción química obedece a una dinámica no lineal y compleja por lo que para poder realizar un buen control de este proceso, la teoría clásica ha resultado insuficiente y es necesaria la aplicación de otras tecnologías como la de redes neuronales artificiales que es la que se utiliza en este trabajo.

La aplicación de las redes neuronales artificiales permite obtener altas velocidades de procesamiento como resultado de las conexiones de sus elementos en forma masiva y en paralelo. Estas características se deben principalmente a la representación distribuida de los datos dentro de la red, y a su capacidad de adaptación y "aprendizaje", para mejorar su desempeño.

Las redes neuronales artificiales, constituyen una de las áreas de la inteligencia artificial que ha despertado mayor interés en los últimos años y constituyen una herramienta sumamente general para abordar una amplia clase de problemas de identificación y control de sistemas dinámicos (Narendra, K.S. y Parthasarathy, K. 1990). En particular, este enfoque resulta muy ventajoso cuando se trata de la identificación de sistemas no lineales (como es el caso de todos los procesos industriales), donde la mayoría de los métodos clásicos no conducen a resultados aceptables, la razón de esto, es que las redes neuronales son capaces de resolver problemas cuya solución por otros métodos convencionales resulta extremadamente difícil. Una de las principales aplicaciones de las redes neuronales en la Ingeniería de Control y Automatización está en su capacidad de identificar sistemas dinámicos por constituir un instrumento muy general y potente para modelar procesos industriales de cualquier grado de complejidad.

El objetivo de este trabajo de investigación es controlar óptimamente la concentración de dióxido de carbono en la columna de absorción empacada del Laboratorio de Operaciones y Procesos Unitarios de la Facultad de Ingeniería Química mediante un modelo de red neuronal artificial.

\section{MATERIALES Y MÉTODOS}

Equipo experimental

Columna de vidrio pyrex empacada con anillos Raschig de vidrio. Circuito de gas comprimido de
$\mathrm{CO} 2$ y aire (cilindro metálico de CO2 con regulador de presión y compresora), un rotámetro para gases. Circuito para la solución de $\mathrm{NaOH}$; el sistema está constituido por un depósito de alimentación, una bomba centrífuga de $1.2 \mathrm{HP}$, tuberías de acero inoxidable, un rotámetro para líquidos.

Además se cuentan con los siguientes instrumentos de medición y control:

Un transmisor de dióxido de carbono serie GMT220. Manómetros MB 1/4 y 0-70 milibares.

Un rotámetro de líquidos de 0-4 Lpm.

Un rotámetro de gases de 0-50 Lpm.

Un variador de velocidad Altivar.

Un controlador de Automatización Programable, CFP 2120 Compact Field Point.

Un computador con el software LabVIEW.

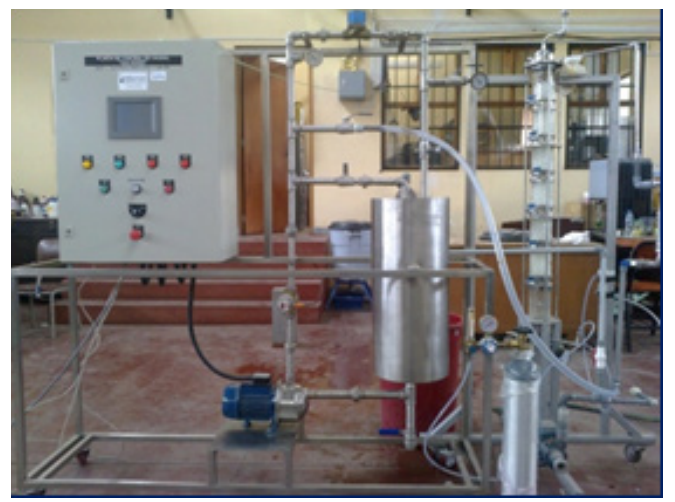

Figura 1. Columna de absorción, LOPU - FIQ.

\section{Modelo de red neuronal artificial}

El modelo de red neuronal artificial implementado es el perceptrón multicapa ya que se ha demostrado ser el aproximador universal de cualquier función no lineal. Tiene una capa de entrada, una capa escondida y una capa de salida $(3-25-1)$, tal como se muestra en la figura 2.

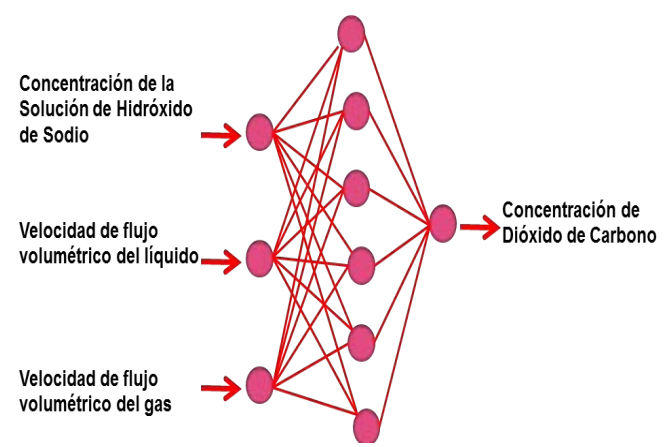

Figura 2. Modelo de red neuronal artificial

Páginas 11-15 
La función de activación para la primera capa es sigmoidal y para la segunda capa es lineal. Las entradas son la concentración de la solución de hidróxido de sodio, la velocidad de flujo volumétrico del líquido y la velocidad de flujo volumétrico del gas. La salida es la concentración de dióxido de carbono.

Para el neurocontrolador se utilizó el modelo inverso (1 - 25 - 3). La función de activación para la primera capa es sigmoidal y para la segunda capa es lineal. La entrada es la concentración de dióxido de carbono y las salidas son la concentración de la solución de hidróxido de sodio, la velocidad de flujo volumétrico del líquido y la velocidad de flujo volumétrico del gas.

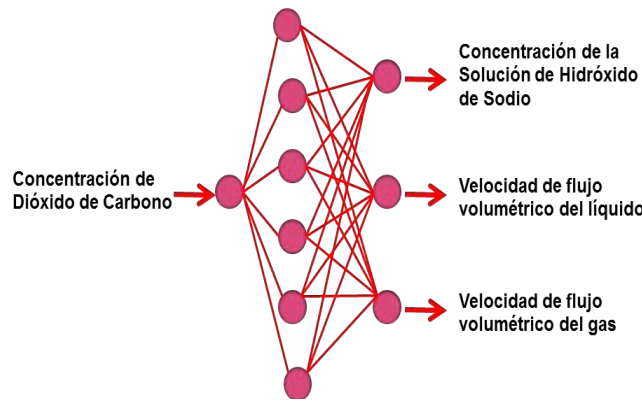

Figura 3. Modelo inverso de red neuronal artificial

El algoritmo implementado para el entrenamiento o aprendizaje de la red neuronal artificial es el de retropropagación, que es una técnica específica para implementar el método del gradiente descendente en un espacio de pesos, para una red multicapa. El algoritmo se encarga de modificar los pesos de cada una de las neuronas de tal manera que la respuesta de la red ante un determinado patrón de entrada se encuentre lo más cerca posible de una salida predeterminada. Para alcanzar este objetivo es necesario definir una función de error que mida el grado de aproximación de cada una de las salidas a los valores que para ellas se desea. Se define así el error de cada neurona de salida como la diferencia entre el valor que se espera tenga cuando se presenta en la entrada el patrón y el que realmente tiene. El error de la salida se calcula considerando el de todas las neuronas que forman esa capa. La forma más habitual de hacerlo es mediante el error cuadrático medio. El objetivo del algoritmo de aprendizaje es minimizar el valor de esa función variando de forma adecuada los pesos de todas las neuronas de la red. Como es una estructura multicapa para la red y el error se calcula únicamente a partir de las salidas de las neuronas de la última capa, es necesario definir un mecanismo que establezca una relación entre la variación de los pesos y esa función, lo que implica una propagación "hacia atrás" del error. Para el entrenamiento, los datos experimentales fueron obtenidos en los rangos adecuados de operación de la columna de absorción para evitar que sucedan los regímenes hidrodinámicos de inundación y canalización. Luego, utilizando el esquema de control por modelo inverso y con el neurocontrolador entrenado y validado se realizaron otras pruebas experimentales para controlar la composición de dióxido de carbono en la columna de absorción empacada.

\section{RESULTADOS}

Para evaluar la habilidad predictiva del modelo inverso de red neuronal artificial se construyó una gráfica de los valores calculados del flujo de solvente versus los valores experimentales del flujo de solvente y se hizo un análisis de regresión lineal. Los valores de los parámetros obtenidos se compararon con los valores ideales: coeficiente de correlación R2 $=1$, pendiente $m=1$ e intercepto $b=0$.

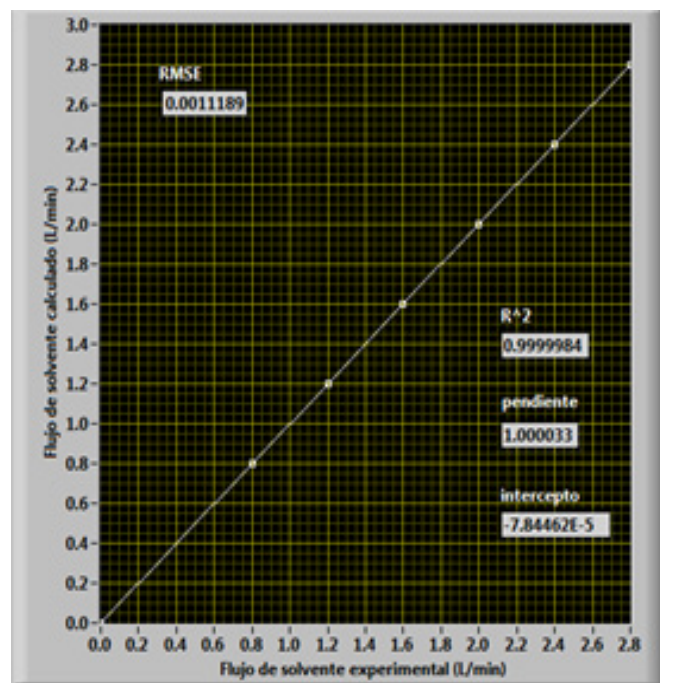

Figura 4. Gráfico de la evaluación de la habilidad predictiva del modelo inverso de la red neuronal artificial

Los parámetros obtenidos del coeficiente de correlación R2 = 0.9999984, pendiente $m=1.000033$ e intercepto $b=-7.84462 \mathrm{E}-5$ se aproximan a los valores de los parámetros ideales, por lo que se deduce que el modelo inverso de red neuronal artificial también tiene buena habilidad predictiva.

Resultados del control neuronal por modelo inverso Una vez entrenada y validada la red se realizaron pruebas del control de la concentración de $\mathrm{CO} 2$ en la columna para diferentes valores de referencia tal como se muestra a continuación: 


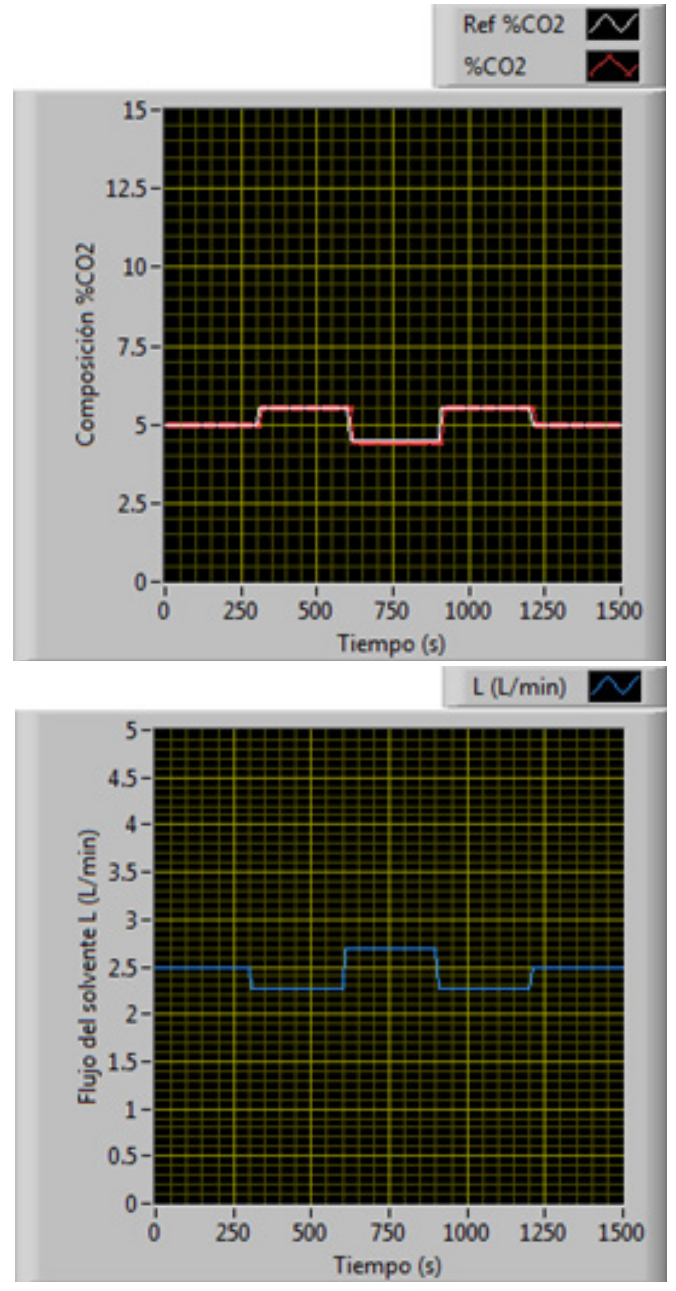

Figura 5. Control de la concentración de $\mathrm{CO} 2$ (solvente $\mathrm{NaOH} 0,11 \mathrm{M}$ )

\section{DISCUSIÓN}

Para evaluar la respuesta del neurocontrolador se realizaron varias pruebas durante 25 minutos aproximadamente (1500 segundos).

En la figura 5, se observa que en tiempo igual a 0 segundos parte de una concentración de $5.0 \%$ de $\mathrm{CO} 2$, luego en tiempo igual a 300 segundos se cambió el valor de referencia de $5.0 \%$ a $5.5 \%$ de $\mathrm{CO} 2$, notándose que el neurocontrolador logró mantener la concentración del $\mathrm{CO} 2$ alrededor del valor de referencia deseado. En tiempo igual a 600 segundos se realizó otro cambio en el valor de referencia pasando de $5.5 \%$ de $\mathrm{CO} 2$ a $4.5 \%$ de $\mathrm{CO} 2$ consiguiendo también que el neurocontrolador mantenga la concentración del $\mathrm{CO} 2$ cerca al nuevo valor de referencia. Luego, en tiempo igual a 900 segundos se hizo un nuevo cambio en el valor de referencia de $4,5 \%$ de $\mathrm{CO} 2$ a $5,5 \%$ de $\mathrm{CO} 2$ consiguiéndose asimismo buen acercamiento al valor de referencia deseado. Finalmente en tiempo igual a 1200 segundos se hizo un último cambio en el valor de referencia pasando de $5,5 \%$ de $\mathrm{CO} 2$ a $5,0 \%$ de $\mathrm{CO} 2$, en este caso también el neurocontrolador logró mantener la concentración de $\mathrm{CO} 2$ en el nuevo valor de referencia. En esta figura también se muestra la evolución de la variable manipulada que es el flujo del solvente $\mathrm{NaOH}$ para conseguir los resultados de control deseados.

Esta investigación llego a las siguientes conclusiones:

1. Se implementó el modelo de red neuronal artificial de la columna de absorción empacada y del neurocontrolador.

2. Se realizó el entrenamiento y la validación del modelo de red neuronal artificial de la columna de absorción empacada y del neurocontrolador.

3. Se controló óptimamente la concentración de dióxido de carbono en la columna de absorción empacada del Laboratorio de Operaciones y Procesos Unitarios mediante el neurocontrolador (modelo inverso de la red neuronal artificial).

\section{REFERENCIAS}

Bhat N. V., McAvoy T. J. (1990). Use of Neural Nets for Dynamic Modeling and Control of Chemical Process Systems. J. Computers Chem. Engng. Vol. 14, No. 4/5: 573-583.

Bhat N. V., McAvoy T. J. (1992). Determining Model Structure for Neural Models by Network Stripping. J. Computers Chem. Engng. Vol. 16, No. 4: 271281.

Di Massimo C., Montague G. A., Willis M. J., Tham M. T., Morris A. J. (1992). Towards Improved Penicilin Fermentation via Artificial Neural Networks. J. Computers Chem. Engng. Vol. 16, No. 4: 283291.

Hagan M. T., Demuth H. B., Beale M. (1996). Neural Networks Design, United States of America: PWS Publishing Company.

Hoff K.A. (2003). Modeling and Experimental study of carbon dioxide absorption in a membrane contactor. Norwegian University of Science and Technology.

Hoskins J. C., Himmelblau D. M. (1988). Artificial Neural Networks Models of Knowledge Representation in Chemical Engineering. J. Computers Chem. Engng. Vol. 12, No. 9: 881890.

Lin-Chen. (2007). Carbon dioxide into $\mathrm{NaOH}$ solution 
in a Cross-Flow Rotating Packed Bed. J. Ind. Eng. Chem. Vol. 13.

Narendra K. S., Parthasarathy K. (1990). Identification and Control of Dynamical Systems Using Neural Networks. J. IEEE Transactions on Neural Networks. Vol. 1, No. 1: 4-27.

Olutoye M. A., Mohammed A. (2006). Modelling of a Gas-Absorption Packed Column for Carbon Dioxide-Sodium Hydroxide System. AU J.T. 10(2): 132-140.

Psichogios D. C., Ungar L. H. (1991). Direct and Indirect Model Based Control using Artificial Neural Networks. J. Ind. Engng. Chem. Res. Vol. 30, 2564-2573.

Sánchez Camperos E., Alanis García A. (2006). Redes neuronales. Pearson, Prentice Hall.

Soloway D. (1996). Neural Generalized Predictive Control. Dearborn: Proceeding IEEE International symposium on Intelligent Control. 277-282.

Temeng K. O., Schnelle P. D., McAvoy T. J. (1995). Model Predictive Control of an Industrial Packed Bed Reactor using Neural Networks. J. Proc. Control. Vol. 5, No. 1: 19-27.

Willis M. J., Montague G. A., Di Massimo C., Tham M. T., Morris A. J. (1992). Artificial Neural Networks in Process Estimation and Control. J. Automatica. Vol. 28, No. 6: 1181-1187.

Yeh-Pennline. (1999). Study of CO2 absorption and desorption in a packed column. U.S. Department of Energy. 